\title{
Imaging Neuromodulatory Signaling Events at Single Cell Resolution in Behaving Animal
}

\author{
Lei $\mathrm{Ma}^{1}$, Bart C. Jongbloets ${ }^{1}$, Wei-Hong Xiong ${ }^{1}$, Haining Zhong ${ }^{1}$ and Tianyi Mao ${ }^{1, *}$ \\ 1. Vollum Institute, Oregon Health \& Science University, Portland, OR, USA. \\ * Corresponding author: mao@ohsu.edu
}

Genetically encoded fluorescence indicators, such as the GCaMP family calcium indicators, in combination with the advancement of microscopy, have revolutionized many fields in biomedical research. They allow for simultaneous in vivo recording of a large number of cells with genetically defined cell types, and linking cellular events to animal behavior [1]. In this meeting, we will present our work in the development of genetically encoded indicators for monitoring intracellular events downstream of neuromodulation, and implementing in vivo imaging of these indicators in combination with two-photon fluorescent lifetime imaging microscopy (2pFLIM) [2].

In the brain, there are two primary modes of chemical communication between neurons: fast synaptic transmission, such as that mediated by glutamate and GABA, and slow synaptic transmission, such as that mediated by norepinephrine (NE), dopamine (DA), serotonin, and opioids [3]. Slow synaptic transmission, also known as neuromodulation, regulates the excitability, synaptic plasticity and other essential aspects of neuronal function. While great strides have been made in improvement of multielectrode recording, voltage imaging and calcium imaging, allowing for examining the dynamics of neuronal electrical activities that are controlled directly by fast synaptic transmission in behaving animals, little is known about the precise neuromodulatory events that occur in living animals since the imaging modality to reliably record the relevant activities triggered by neuromodulation at single cell resolution in vivo is not well established. The cyclic AMP (cAMP)/protein kinase A (PKA) pathway is a common downstream signal transduction pathway for the major neuromodulators [4]. Imaging the activity of the cAMP/PKA pathway in individual neurons, therefore, provides readout for neuromodulatory events in large neuronal population, in analogous to imaging calcium for fast transmission events.

Although genetically encoded cAMP/PKA indicators have been used in vitro [5], their applications under the more challenging in vivo imaging conditions have been difficult. We choose to image and optimize the indicators using 2pFLIM for two considerations. First, because 2pFLIM measures only the lifetime of the donor in a FRET fluorophore pair, it does not suffer from wavelength-dependent light scattering, and provides stable measurements across different tissue depths. Second, 2pFLIM measurements only occurs in one color channel $[6,7]$. In conjunction with the use of a low quantum yield acceptor, this frees the other color channel for multiplexed measurement of orthogonal neuronal properties (e.g., simultaneous imaging of a calcium indicator or a morphological marker). We started by screening for the best existing PKA indicators [8] for their responses to NE in organotypic slice culture preparations that have the potential to inform the indicators' performance in vivo (Figure 1). By using subcellular targeting strategy, we further developed a variant (named tAKAR $\alpha$ ) with much increased sensitivity and a broadened dynamic range (Figure 2). This indicator enabled the detection of PKA activation by $\mathrm{NE}$ at physiologically-relevant concentrations and kinetics, and by optogenetically released endogenous DA. In vivo longitudinal 2pFLIM imaging of tAKAR $\alpha$ tracked bidirectional PKA activities in individual neurons in awake mice, and revealed that locomotion elicits cell-specific neuromodulator 
PKA responses (Figure 3). Our data suggest that tAKAR $\alpha$, combined with 2pFLIM enables the interrogation of neuromodulation-induced PKA signaling in behaving animals [9].

References:

[1] MZ Lin and MJ Schnitzer, Nat Neurosci. 19 (2016), p. 1142.

[2] L Ma et al., Neuron 99 (2018), p. 665.

[3] P Greengard, Science 294 (2001), p. 1024.

[4] SH Francis and JD Corbin, Annu Rev Physiol. 56 (1994), p. 237.

[5] J Zhang et al., Proc Natl Acad Sci U S A 98 (2001), p. 14997.

[6] R Yasuda, Curr Opin Neurobiol. 16 (2006), p. 551.

[7] G Yellen and R Mongeon, Curr Opin Chem Biol. 27 (2015), p. 24.

[8] Y Chen et al., Neuron 96 (2017), p. 1070.

[9] This work was supported by NIH grants R01NS081071, U01NS094247, and R01NS014944.

A

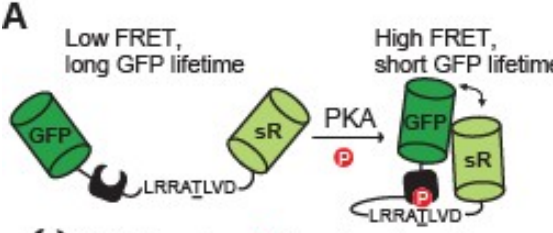

4 FHA1 domain eThreonine phosphate

B

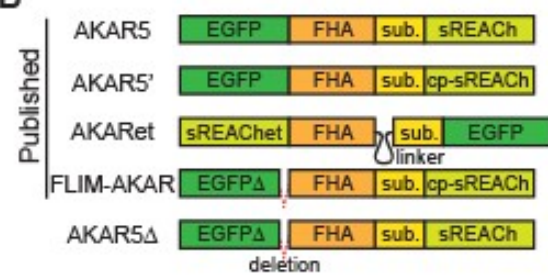

C
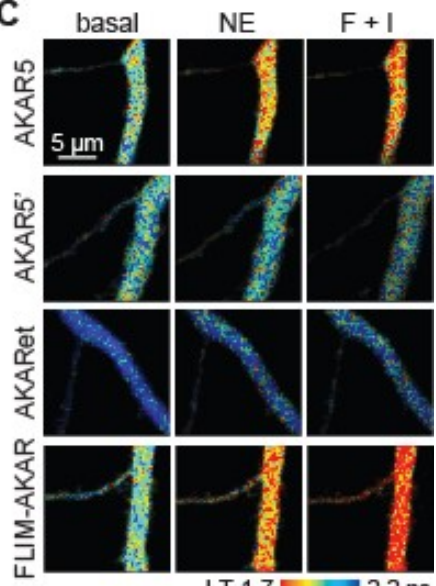
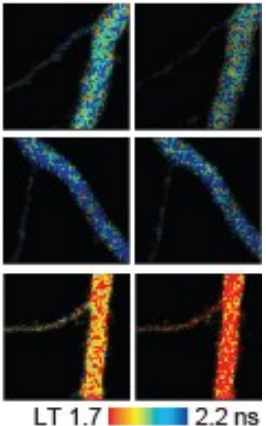

Figure 1. PKA indicator design and assays for screening existing indicators.

A

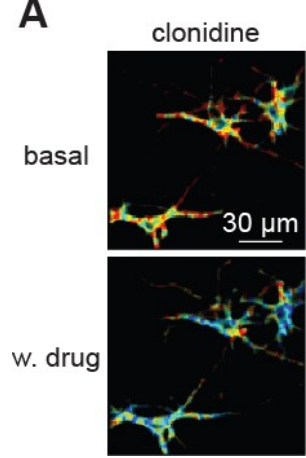

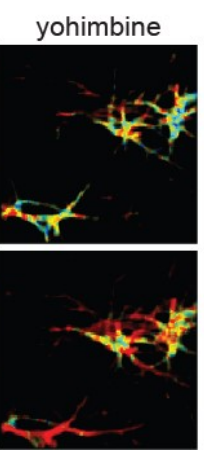

B

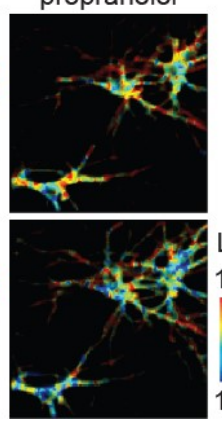

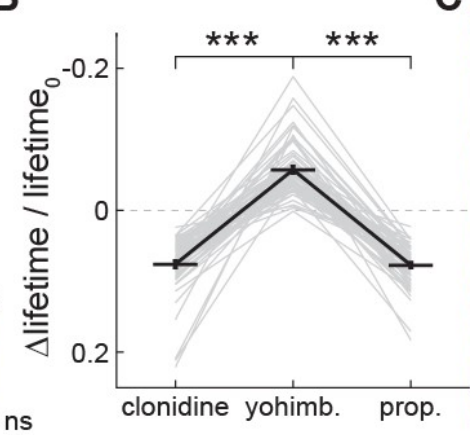
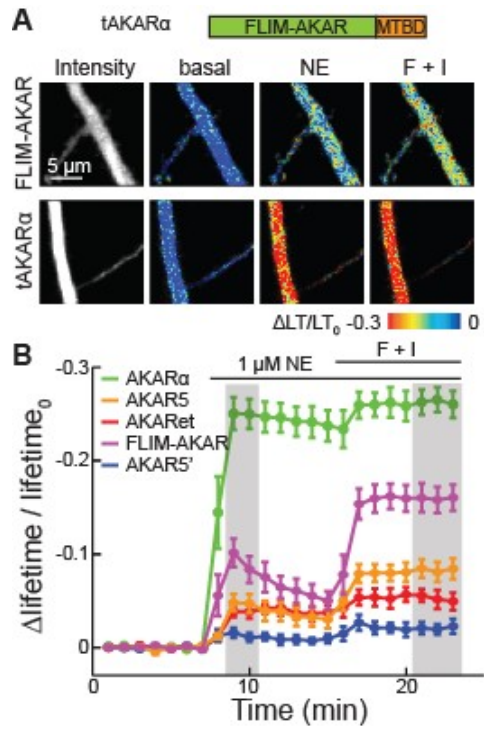

Figure 2. Design of AKAR $\alpha$ and enhances performance in vitro.

Figure 3. tAKAR $\alpha$ tracked bidirectional PKA activities in individual neurons in awake mice and population responses to locomotion. (A, B) Representative lifetime images (A) and summary results (B) of neurons responding to the indicated pharmacological manipulations in awake mice. $\mathrm{n}=63$ neurons from 6 mice. (C) The $\Delta$ lifetime response timecourses averaged for each of 29 neurons (each row represents a neuron, and each column represents a time point) that are clustered into three groups (marked by dashed lines). 